\title{
FLAURA strikes again: efficacy of osimertinib is independent of PD-L1 expression
}

\author{
Pamela Abdayem^, David Planchard \\ Department of Medical Oncology, Gustave Roussy, Villejuif, France \\ Correspondence to: David Planchard, MD. Department of Medical Oncology, Gustave Roussy, 114 Edouard Vaillant Street, 94805 Villejuif Cedex, \\ France. Email: david.planchard@gustaveroussy.fr. \\ Comment on: Brown H, Vansteenkiste J, Nakagawa K, et al. Programmed Cell Death Ligand 1 Expression in Untreated EGFR Mutated Advanced \\ NSCLC and Response to Osimertinib Versus Comparator in FLAURA. J Thorac Oncol 2020;15:138-43.
}

Submitted May 01, 2020. Accepted for publication Jun 10, 2020.

doi: $10.21037 /$ tlcr-20-628

View this article at: http://dx.doi.org/10.21037/tlcr-20-628

Osimertinib, a third generation tyrosine kinase inhibitor (TKI) of epidermal growth factor receptor (EGFR), achieved impressive results in first-line treatment of patients with metastatic non-small cell lung cancer (NSCLC) with sensitizing EGFR mutations (1). Numerically, with a median progression-free survival (PFS) and overall survival (OS) of 18.9 and 38.6 months respectively, osimertinib surpasses outcomes of all available treatments in metastatic NSCLC including those of immune checkpoint inhibitors (ICIs) $(2,3)$. However, unlike with ICIs, responses are not durable and disease eventually progresses. Outrunning osimertinib in first-line treatment of metastatic EGFR mutation (EGFRm)-positive NSCLC would require novel strategies such as exploring and exploiting the immune environment of EGFR driven disease.

Of 231 tissue blocks available from the screened population in the FLAURA trial, 197 with sufficient tissue were stained for programmed death ligand 1 (PD-L1) expression, using the SP263 Ventana immunohistochemical assay based on tumor cell staining (TC). Immune cell (IC) scoring incorporated into the VENTANA PD-L1 (SP142) assay, was also performed as part of an exploratory analysis. In a recent paper published in the Fournal of Thoracic Oncology, Brown and colleagues demonstrated that the efficacy of osimertinib in first-line treatment of EGFRmpositive metastatic NSCLC was unaffected by PD-L1 expression regardless of the selected staining threshold (1\%, $25 \%$ or $50 \%$ ) and the type of immunohistochemical staining assay (TC or TC and IC). Moreover, EGFR mutations and PD-L1 expression were not mutually exclusive; yet, the prevalence of PD-L1 expression was lower in EGFRmpositive tumors and the difference was more accentuated at higher PD-L1 TC thresholds ( $51 \%$ vs. $68 \%$ at a TC $\geq 1 \%$, $8 \%$ vs. $35 \%$ at $\mathrm{TC} \geq 25 \%$ and $5 \%$ vs. $28 \%$ at TC $\geq 50 \%$ ). In EGFRm-positive patients who received osimertinib $(\mathrm{N}=54)$, median PFS and response rates $(\mathrm{RR})$ matched those obtained in the overall FLAURA population (median PFS 18.9 months, response rate $80 \%$ ) and were unaffected by PD-L1 expression (TC/IC $\geq 1 \%$ versus TC/IC $<1 \%$ for PFS and TC $\geq 1 \%$ versus TC $<1 \%$ for RR) (4).

The key message in Brown et al.'s analysis of the FLAURA population is that the efficacy of osimertinib remains independent of PD-L1 expression which is concordant with Tang et al. and Cho et al.'s findings (4-6). A few studies reported improved survival endpoints (again endpoints were heterogeneous across studies) in EGFRmpositive patients who were also PD-L1 expressers $(7,8)$. Other studies found adverse outcomes in PD-L1 expressers $(9,10)$. All the studies that tried to answer this pending question were retrospective. PD-L1 status was mainly assessed by immunohistochemistry (IHC); yet thresholds varied significantly across studies. Most of these studies were monocentric and used either erlotinib or gefitinib. Data is scarce regarding the interplay between osimertinib, a more potent EGFR TKI, and PD-L1 expression. EGFR T790 mutant NCI-H1975 cell lines treated with

$\wedge$ ORCID: 0000-0002-4477-3633. 
osimertinib had a reduced PD-L1 expression regardless of cell death (11). Osimertinib both induced PD-L1 degradation by proteasomes and reduced PD-L1 mRNA expression (11). The consequence of osimertinib-modulated PD-L1 downregulation is unclear and requires further in vivo studies. ATHENE (NCT03029858) is an ongoing observational study that measures $\mathrm{PD}-\mathrm{L} 1$ value change by TC/IC staining as well as PD-L1 expression positive rate change from the baseline to progressive disease in patients with advanced NSCLC with confirmed T790 mutation after prior EGFR-TKI treatment.

Even though they are not mutually exclusive, epidemiological studies found a lower PD-L1 expression in EGFR-driven tumors. In a pooled analysis of 18 studies with 3,969 patients, Soo et al. obtained a $41 \%$ lower likelihood of PD-L1 expression in EGFRm-positive patients (12). Despite the heterogeneity of study designs and PD-L1 testing assays, these results were corroborated by three additional analyses (13-15). In the FLAURA trial, EGFRm-positive patients with PD-L1 expression $\geq 1 \%$ ( $\mathrm{N}=52)$ were more likely to be of Asian ethnicity (37\% vs. $20 \%$ in PD-L1 negative patients) and to harbor L858R mutations ( $42 \%$ vs. $33 \%$ ). Conversely, non-expressers of PD-L1 were more likely to be of white Caucasian ethnicity (80\% vs. $63 \%$ in PD-L1 expressers) and to harbor exon 19 deletions (63\% vs. 56\%). Smoking status did not vary between the two groups (4). However, these data are merely observational as no multivariate analysis was performed. The majority of related studies evaluate the association between PD-L1 expression and clinicopathologic features including EGFR mutations but provide very little information about the characteristics of PD-L1 expressers among EGFRmpositive patients $(14,16)$. Therefore, characterization of this group of EGFRm-positive patients who are also PDL1 expressers is pertinent and might help identify which patients benefit from ICIs after EGFR-TKIs. Interestingly, in a study of 25 EGFRm-positive patients who received nivolumab after disease progression on EGFR-TKI therapy, Haratani et al. observed a higher PD-L1 expression in T790M-negative versus T790M-positive patients (17).

The activation of the PD-1/PD-L1 pathway negatively regulates immune responses and allows for cancer progression and metastasis. PD-L1 expression level is currently used as a predictive biomarker for anti-PD-1/PDL1 ICIs in the treatment of NSCLC (18). However, this biomarker is far from perfect and did not predict response to first-line nivolumab in stage IV or recurrent NSCLC (19). Furthermore, a phase II trial of pembrolizumab in TKI- naïve EGFRm-positive and PD-L1 positive (PD-L1 $\geq 1 \%$ ) patients with advanced NSCLC was prematurely ceased due to lack of efficacy despite a PD-L1 expression $\geq 50 \%$ in $73 \%$ of patients (20). Indeed, EGFR mutated tumors do not seem to respond well to immunotherapy. The biological basis for the lack of efficacy remains unclear and the majority of the trials testing the efficacy of ICIs excluded patients with driver mutations $(2,21)$. Conversely, IMpower 150 was the first randomized phase 3 trial of an ICI combined with chemotherapy and bevacizumab that demonstrated improved PFS and OS in patients with sensitizing EGFR mutations who were previously treated with EGFR-TKIs for advanced non-squamous NSCLC (22). Table 1 lists the main studies of ICIs in advanced NSCLC that included EGFRm-positive patients (3,20,22-32). CheckMate 722 and Keynote-789 are 2 ongoing studies comparing the combination of chemotherapy with Nivolumab (with or without ipilimumab) or Pembrolizumab, respectively, to chemotherapy alone in TKI-resistant advanced nonsquamous EGFRm+ NSCLC (NCT02864251 and NCT03515837). Additional information on post-study ICI use in Brown et al.'s analysis of FLAURA would have been particularly instructive. PD-L1 might not be the best biomarker to predict the efficacy of immunotherapy in EGFRm-positive tumors. Other biomarkers were studied in NSCLC, namely the tumor mutational burden (TMB) measured as the number of mutations in the whole exome or per megabase and the tumor microenvironment (TME). TMB was a strong predictor of PFS irrespective of PDL1 expression in a phase III trial combining nivolumab and ipilimumab as first-line treatment of advanced NSCLC without driver mutations (21). A significant association between TMB and EGFRm-negative status $(\mathrm{P}=0.0111)$ was noted in a multivariate analysis (33). On the other hand, Gainor et al. found that a low expression of PD-L1 and CD8 positive tumor infiltrating lymphocytes (TILs) within the TME might explain the disappointing results of immunotherapy in EGFRm-positive tumors (34). In opposite, type 1 TME phenotype with both high PD-L1 and CD8 positive TILs might be a promising predictor of efficacy of anti-PD-1/PD-L1 therapy in NSCLC including EGFRm-positive disease (10,35).

Besides selecting the adequate population and finding the correct predictive biomarker, combination therapy might allow ICIs to find their place in EGFR-driven disease. Studies suggested an active role for the EGFR oncogene in remodeling the TME. EGFRm-positive tumors may be characterized by host $\mathrm{T}$ cell exhaustion specifically through 


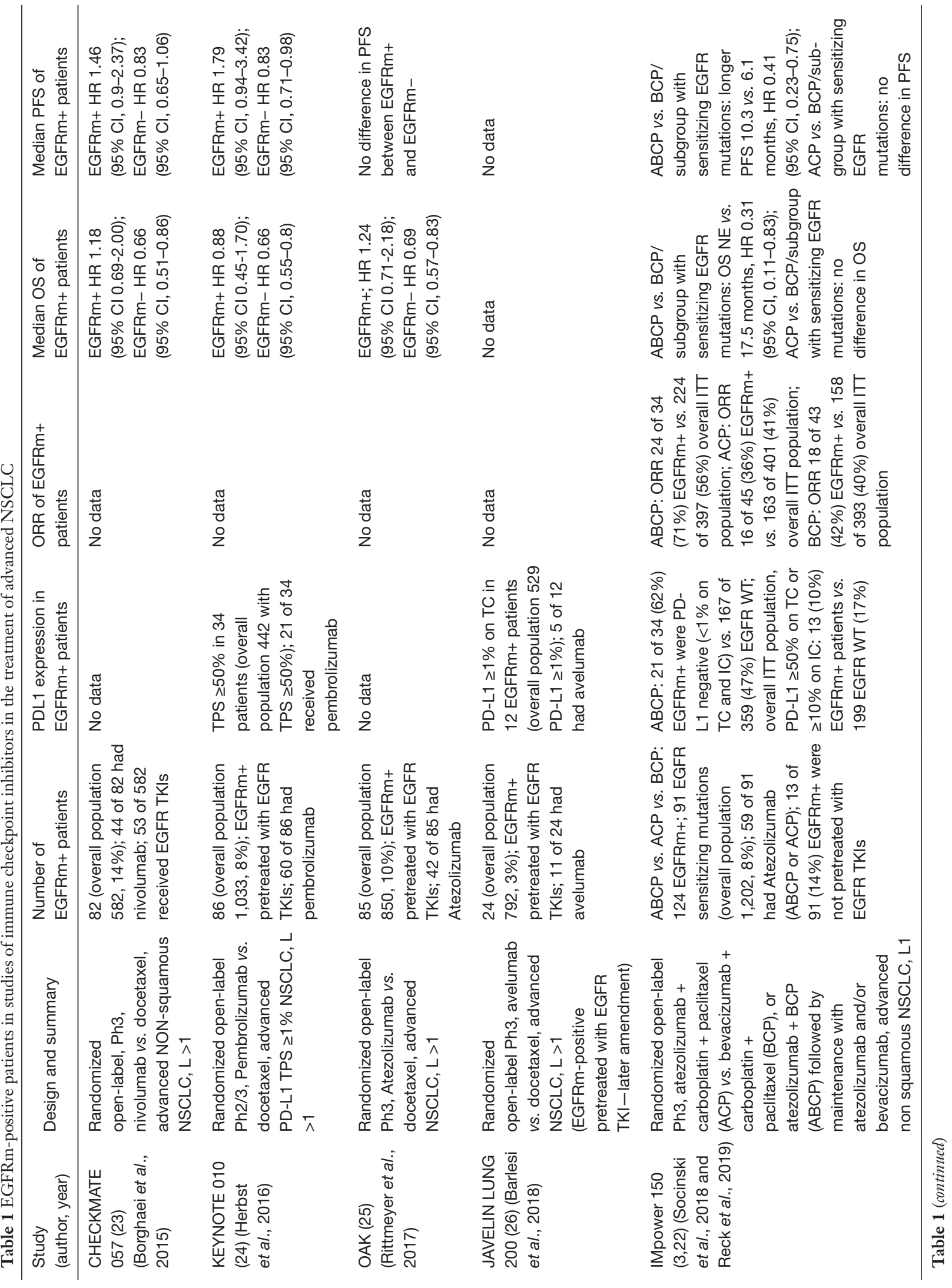




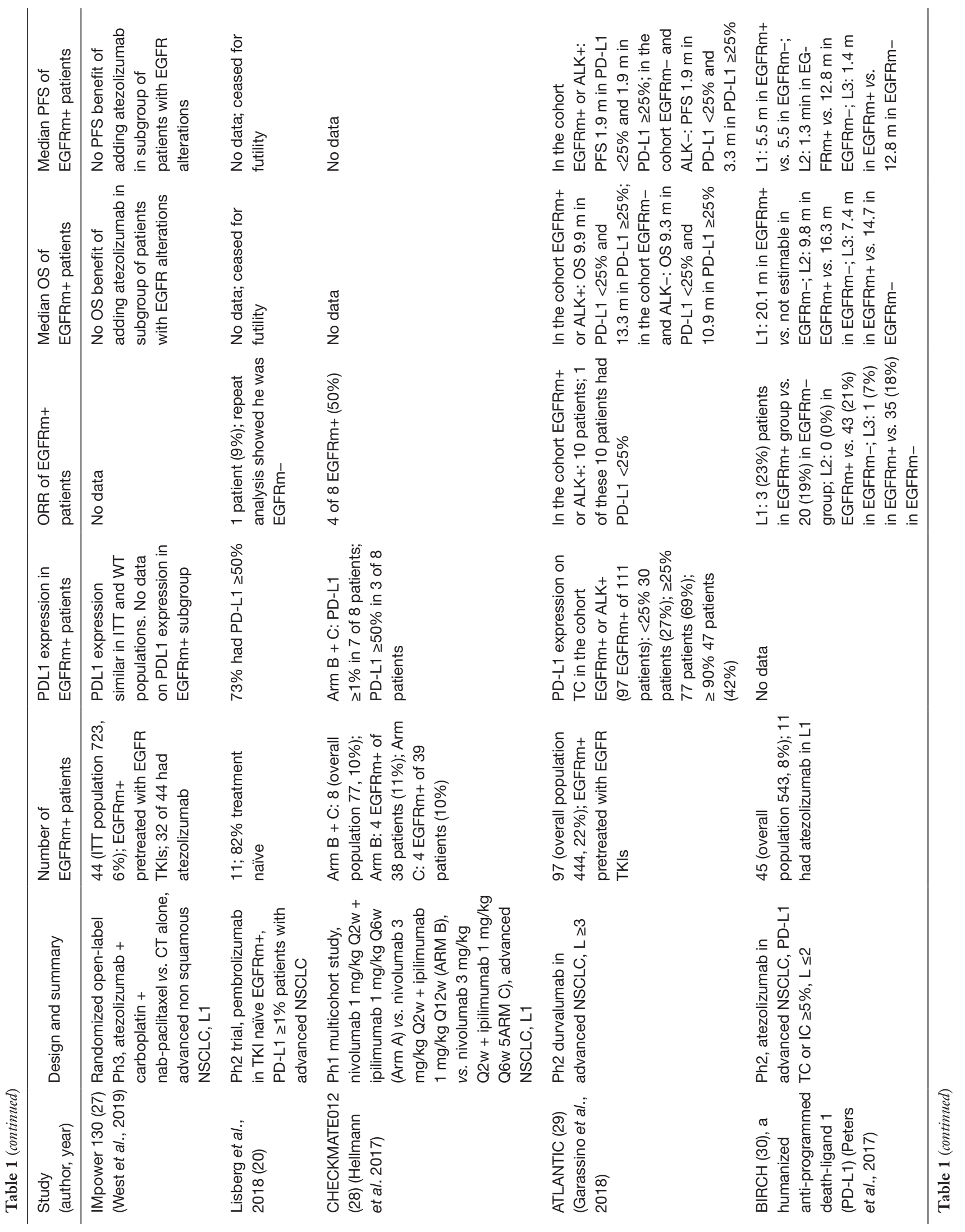




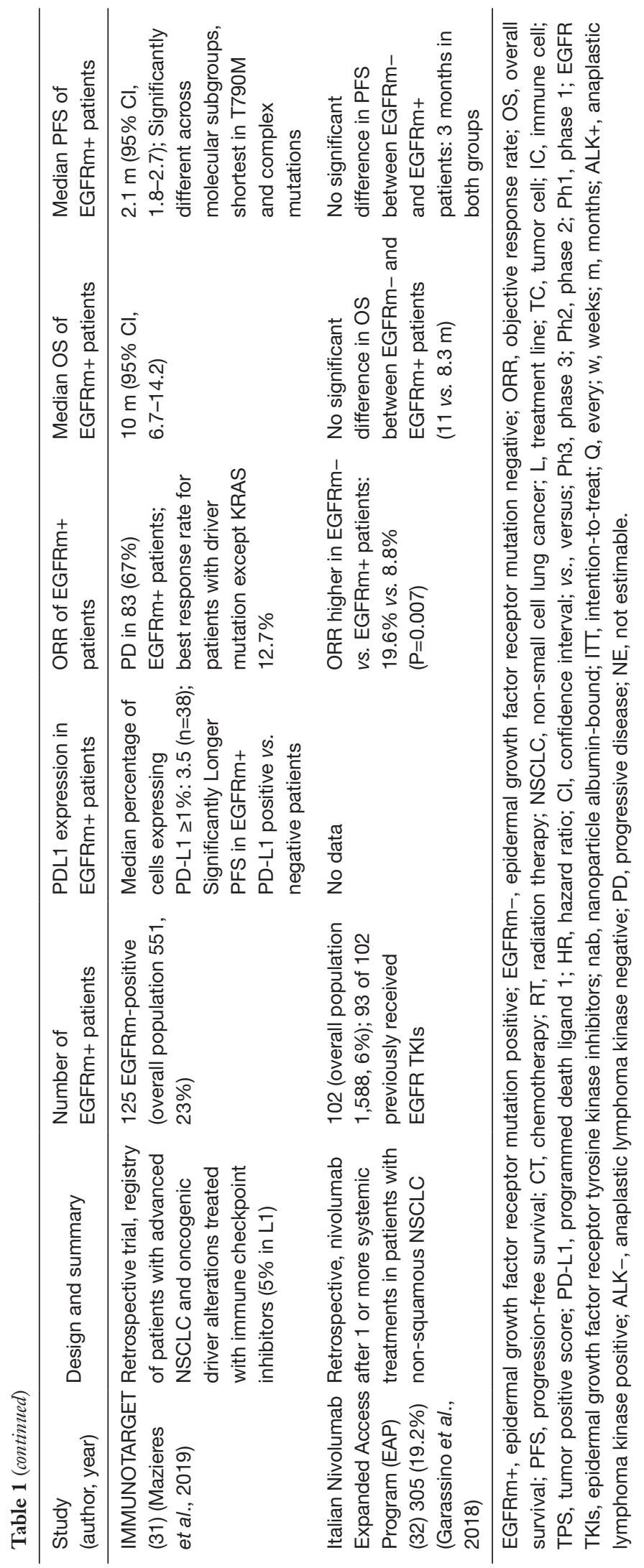


upregulation of the PD-1/PD-L1 pathway (36). In addition, EGFR TKIs have contrasting immunomodulatory effects. Erlotinib and Gefitinib increase the sensitivity of lung cancer cells to natural killer cell-mediated lysis through upregulation of NKG2D ligands (37). They also enhance major histocompatibility index class I (MHCI) and II (MHCII) molecules in response to IFN- $\gamma$ thus increasing $\mathrm{T}$ cell mediated tumor killing (38). Conversely, EGFR TKIs also have immunosuppressive effects through inhibition of T-cell proliferation and activation and through inhibition of monocyte differentiation and increase in circulating myeloid derived suppressor cells (39). Akbay et al. found a reduction in PD-L1 expression in vitro after treatment with EGFR TKIs (36). Based on the previous findings of immunostimulatory and immunosuppressive functions of EGFR TKIs, one can conclude that the addition of these drugs to ICIs might not be the miraculous solution to implement immunotherapy in EGFRm-positive advanced NSCLC. Moreover, EGFR TKIs and ICIs have overlapping toxicities such as pneumonitis, which could be life-threatening in some cases, thus caution is advised when designing phase I combination studies. In fact, Schoenfeld et al. reported severe immune-related adverse events in $15 \%$ of patients treated with sequential ICIs followed by osimertinib, especially when the latter was started within the first 3 months following ICIs (40). Also in the multi-arm phase Ib TATTON trial, the combination of osimertinib and durvalumab in patients with EGFRm-positive advanced NSCLC progressing on prior EGFR TKI treatment was associated with interstitial lung disease in $22 \%$ of patients including one with a grade 5 pneumonia (41). On the other hand, yes-associated protein (YAP) is the main mediator of the Hippo signalling pathway. Its activation through loss of Hippo signalling by mutation, and downregulation of the core Hippo components, promotes cancer progression, drug resistance and metastasis in NSCLC. The EGFR/MAPK signalling pathway stimulates YAP. Inhibition of YAP could be used in the treatment of NSCLC with acquired resistance to EGFR-TKIs, and to increase sensitivity to BRAF and MEK inhibitors $(42,43)$. Anti YAP therapy could also be combined with ICIs to improve their efficacy in EGFR driven disease. Statins, cucurbitacin E, dasatinib, dobutamine, norcantharidin, JQ1, agave and MLN8237 are some of the molecules that inhibit YAP and that could be tested in combination with immunotherapy in future phase I studies of EGFR driven, TKI resistant tumors (44).

Despite the magnitude and the solid design of FLAURA, the debate about oncogene addiction and immune escape is far from being closed. Outrunning osimertinib in the first-line treatment of EGFRm-positive advanced NSCLC requires us to deepen our knowledge of the immune microenvironment of EGFR-driven disease, to find consistent prognostic and predictive biomarkers and finally to carefully develop both smart and safe combination therapies.

\section{Acknowledgments}

Funding: None.

\section{Footnote}

Provenance and Peer Review: This article was commissioned by the editorial office, Translational Lung Cancer Research. The article did not undergo external peer review.

Conflicts of Interest: Both authors have completed the ICMJE uniform disclosure form (available at http://dx.doi. org/10.21037/tlcr-20-628). PA reports non-financial support from Pierre Fabre, non-financial support from Roche, outside the submitted work. DP reports personal fees from AstraZeneca, personal fees from BMS, personal fees from Boehringer Ingelheim, personal fees from Celgene, personal fees from Daiichi Sankyo, personal fees from Eli Lilly, personal fees from Merck, personal fees from Novartis, personal fees from Pfizer, personal fees from prIME Oncology, personal fees from Peer CME, personal fees from Roche, personal fees from Samsung, outside the submitted work; and Clinical trials research (as principal investigator or co-investigator): AstraZeneca, Bristol-Myers Squibb, Boehringer Ingelheim, Eli Lilly, Merck, Novartis, Pfizer, Roche, Medimmun, Sanofi-Aventis, Taiho Pharma, Novocure, Daiichi Sankyo.

Ethical Statement: The authors are accountable for all aspects of the work in ensuring that questions related to the accuracy or integrity of any part of the work are appropriately investigated and resolved.

Open Access Statement: This is an Open Access article distributed in accordance with the Creative Commons Attribution-NonCommercial-NoDerivs 4.0 International License (CC BY-NC-ND 4.0), which permits the noncommercial replication and distribution of the article with the strict proviso that no changes or edits are made and the original work is properly cited (including links to both the formal publication through the relevant DOI and the license). 
See: https://creativecommons.org/licenses/by-nc-nd/4.0/.

\section{References}

1. Ramalingam SS, Vansteenkiste J, Planchard D, et al. Overall Survival with Osimertinib in Untreated, EGFR-Mutated Advanced NSCLC. N Engl J Med 2020;382:41-50.

2. Reck M, Rodríguez-Abreu D, Robinson AG, et al. Pembrolizumab versus Chemotherapy for PD-L1Positive Non-Small-Cell Lung Cancer. N Engl J Med 2016;375:1823-33.

3. Socinski MA, Jotte RM, Cappuzzo F, et al. Atezolizumab for First-Line Treatment of Metastatic Nonsquamous NSCLC. N Engl J Med 2018;378:2288-301.

4. Brown H, Vansteenkiste J, Nakagawa K, et al. Programmed Cell Death Ligand 1 Expression in Untreated EGFR Mutated Advanced NSCLC and Response to Osimertinib Versus Comparator in FLAURA. J Thorac Oncol 2020;15:138-43.

5. Cho JH, Zhou W, Choi YL, et al. Retrospective Molecular Epidemiology Study of PD-L1 Expression in Patients with EGFR-Mutant Non-small Cell Lung Cancer. Cancer Res Treat 2018;50:95-102.

6. Tang Y, Fang W, Zhang Y, et al. The association between PD-L1 and EGFR status and the prognostic value of PDL1 in advanced non-small cell lung cancer patients treated with EGFR-TKIs. Oncotarget 2015;6:14209-19.

7. D'Incecco A, Andreozzi M, Ludovini V, et al. PD-1 and PD-L1 expression in molecularly selected non-small-cell lung cancer patients. Br J Cancer 2015;112:95-102.

8. Lin C, Chen X, Li M, et al. Programmed Death-Ligand 1 Expression Predicts Tyrosine Kinase Inhibitor Response and Better Prognosis in a Cohort of Patients With Epidermal Growth Factor Receptor Mutation-Positive Lung Adenocarcinoma. Clin Lung Cancer 2015;16:e25-35.

9. Soo RA, Kim HR, Asuncion BR, et al. Significance of immune checkpoint proteins in EGFR-mutant non-small cell lung cancer. Lung Cancer 2017;105:17-22.

10. Su S, Dong ZY, Xie Z, et al. Strong Programmed Death Ligand 1 Expression Predicts Poor Response and De Novo Resistance to EGFR Tyrosine Kinase Inhibitors Among NSCLC Patients With EGFR Mutation. J Thorac Oncol 2018;13:1668-75.

11. Jiang XM, Xu YL, Huang MY, et al. Osimertinib (AZD9291) decreases programmed death ligand-1 in EGFR-mutated non-small cell lung cancer cells. Acta Pharmacol Sin 2017;38:1512-20.

12. Soo RA, Lim SM, Syn NL, et al. Immune checkpoint inhibitors in epidermal growth factor receptor mutant non-small cell lung cancer: Current controversies and future directions. Lung Cancer 2018;115:12-20.

13. Dong ZY, Zhang JT, Liu SY, et al. EGFR mutation correlates with uninflamed phenotype and weak immunogenicity, causing impaired response to PD-1 blockade in non-small cell lung cancer. OncoImmunology 2017;6:e1356145.

14. Zhang M, Li G, Wang Y, et al. PD-L1 expression in lung cancer and its correlation with driver mutations: a metaanalysis. Sci Rep 2017;7:10255.

15. Schoenfeld AJ, Rizvi H, Bandlamudi C, et al. Clinical and molecular correlates of PD-L1 expression in patients with lung adenocarcinomas. Ann Oncol 2020;31:599-608.

16. Ng TL, Liu Y, Dimou A, et al. Predictive value of oncogenic driver subtype, programmed death-1 ligand (PD-L1) score, and smoking status on the efficacy of PD-1/PD-L1 inhibitors in patients with oncogene-driven non-small cell lung cancer. Cancer 2019;125:1038-49.

17. Haratani K, Hayashi H, Tanaka T, et al. Tumor immune microenvironment and nivolumab efficacy in EGFR mutation-positive non-small-cell lung cancer based on T790M status after disease progression during EGFRTKI treatment. Ann Oncol 2017;28:1532-9.

18. Passiglia F, Bronte G, Bazan V, et al. PD-L1 expression as predictive biomarker in patients with NSCLC: a pooled analysis. Oncotarget 2016;7:19738-47.

19. Carbone DP, Reck M, Paz-Ares L, et al. First-Line Nivolumab in Stage IV or Recurrent Non-Small-Cell Lung Cancer. N Engl J Med 2017;376:2415-26.

20. Lisberg A, Cummings A, Goldman JW, et al. A Phase II Study of Pembrolizumab in EGFR-Mutant, PD-L1+, Tyrosine Kinase Inhibitor Naïve Patients With Advanced NSCLC. J Thorac Oncol 2018;13:1138-45.

21. Hellmann MD, Ciuleanu T-E, Pluzanski A, et al. Nivolumab plus Ipilimumab in Lung Cancer with a High Tumor Mutational Burden. N Engl J Med 2018;378:2093-104.

22. Reck M, Mok TSK, Nishio M, et al. Atezolizumab plus bevacizumab and chemotherapy in non-small-cell lung cancer (IMpower150): key subgroup analyses of patients with EGFR mutations or baseline liver metastases in a randomised, open-label phase 3 trial. Lancet 2019;7:387-401.

23. Borghaei H, Paz-Ares L, Horn L, et al. Nivolumab versus Docetaxel in Advanced Nonsquamous Non-Small-Cell Lung Cancer. N Engl J Med 2015;373:1627-39.

24. Herbst RS, Baas P, Kim DW, et al. Pembrolizumab versus docetaxel for previously treated, PD-L1-positive, advanced non-small-cell lung cancer (KEYNOTE-010): a 
randomised controlled trial. Lancet 2016;387:1540-50.

25. Rittmeyer A, Barlesi F, Waterkamp D, et al. Atezolizumab versus docetaxel in patients with previously treated non-small-cell lung cancer (OAK): a phase 3, openlabel, multicentre randomised controlled trial. Lancet 2017;389:255-65.

26. Barlesi F, Vansteenkiste J, Spigel D, et al. Avelumab versus docetaxel in patients with platinum-treated advanced non-small-cell lung cancer (JAVELIN Lung 200): an open-label, randomised, phase 3 study. Lancet Oncol 2018;19:1468-79.

27. West H, McCleod M, Hussein M, et al. Atezolizumab in combination with carboplatin plus nab-paclitaxel chemotherapy compared with chemotherapy alone as firstline treatment for metastatic non-squamous non-smallcell lung cancer (IMpower130): a multicentre, randomised, open-label, phase 3 trial. Lancet Oncol 2019;20:924-37.

28. Hellmann MD, Rizvi NA, Goldman JW, et al. Nivolumab plus ipilimumab as first-line treatment for advanced nonsmall-cell lung cancer (CheckMate 012): results of an open-label, phase 1, multicohort study. Lancet Oncol 2017;18:31-41.

29. Garassino MC, Cho BC, Kim JH, et al. Durvalumab as third-line or later treatment for advanced non-small-cell lung cancer (ATLANTIC): an open-label, single-arm, phase 2 study. Lancet Oncol 2018;19:521-36.

30. Peters S, Gettinger S, Johnson ML, et al. Phase II Trial of Atezolizumab As First-Line or Subsequent Therapy for Patients With Programmed Death-Ligand 1-Selected Advanced Non-Small-Cell Lung Cancer (BIRCH). J Clin Oncol 2017;35:2781-9.

31. Mazieres J, Drilon A, Lusque A, et al. Immune checkpoint inhibitors for patients with advanced lung cancer and oncogenic driver alterations: results from the IMMUNOTARGET registry. Ann Oncol 2019;30:1321-8.

32. Garassino MC, Gelibter AJ, Grossi F, et al. Italian Nivolumab Expanded Access Program in Nonsquamous Non-Small Cell Lung Cancer Patients: Results in NeverSmokers and EGFR-Mutant Patients. J Thorac Oncol 2018;13:1146-55.

33. Ozaki Y, Muto S, Takagi H, et al. Tumor mutation burden and immunological, genomic, and clinicopathological factors as biomarkers for checkpoint inhibitor treatment of patients with non-small-cell lung cancer. Cancer Immunol Immunother 2020;69:127-34.

34. Gainor JF, Shaw AT, Sequist LV, et al. EGFR Mutations and ALK Rearrangements Are Associated with Low
Response Rates to PD-1 Pathway Blockade in Non-Small Cell Lung Cancer: A Retrospective Analysis. Clin Cancer Res 2016;22:4585-93.

35. Liu SY, Dong ZY, Wu SP, et al. Clinical relevance of PD$\mathrm{L} 1$ expression and CD8+ T cells infiltration in patients with EGFR-mutated and ALK-rearranged lung cancer. Lung Cancer 2018;125:86-92.

36. Akbay EA, Koyama S, Carretero J, et al. Activation of the PD-1 pathway contributes to immune escape in EGFRdriven lung tumors. Cancer Discov 2013;3:1355-63.

37. Kim H, Kim SH, Kim MJ, et al. EGFR inhibitors enhanced the susceptibility to NK cell-mediated lysis of lung cancer cells. J Immunother 2011;34:372-81.

38. Pollack BP, Sapkota B, Cartee TV. Epidermal Growth Factor Receptor Inhibition Augments the Expression of MHC Class I and II Genes. Clin Cancer Res 2011;17:4400-13.

39. Savikko J, Rintala JM, Rintala S, et al. Epidermal growth factor receptor inhibition by erlotinib prevents vascular smooth muscle cell and monocyte-macrophage function in vitro. Transpl Immunol 2015;32:175-8.

40. Schoenfeld AJ, Arbour KC, Rizvi H, et al. Severe immune-related adverse events are common with sequential PD-(L)1 blockade and osimertinib. Ann Oncol 2019;30:839-44.

41. Oxnard GR, Yang JCH, Yu H, et al. TATTON: a multi-arm, phase Ib trial of osimertinib combined with selumetinib, savolitinib, or durvalumab in EGFR-mutant lung cancer. Ann Oncol 2020;31:507-16.

42. Lin L, Sabnis AJ, Chan E, et al. The Hippo effector YAP promotes resistance to RAF- and MEK-targeted cancer therapies. Nat Genet 2015;47:250-6.

43. Hsu PC, Jablons DM, Yang CT, et al. Epidermal Growth Factor Receptor (EGFR) Pathway, Yes-Associated Protein (YAP) and the Regulation of Programmed Death-Ligand 1 (PD-L1) in Non-Small Cell Lung Cancer (NSCLC). Int J Mol Sci 2019;20:3821.

44. Hsu PC, Tian B, Yang YL, et al. Cucurbitacin E inhibits the Yes-associated protein signaling pathway and suppresses brain metastasis of human non-small cell lung cancer in a murine model. Oncol Rep 2019;42:697-707.

Cite this article as: Abdayem P, Planchard D. FLAURA strikes again: efficacy of osimertinib is independent of PDL1 expression. Transl Lung Cancer Res 2020;9(5):2165-2172. doi: $10.21037 /$ tlcr-20-628 